\title{
Prevalence and risk factors for type 2 diabetes mellitus with Prader-Willi syndrome: a single center experience
}

\author{
Aram Yang, Jinsup Kim, Sung Yoon Cho* and Dong-Kyu Jin ${ }^{*}$
}

\begin{abstract}
Background: Prader-Willi syndrome (PWS) is often related to severe obesity and type-2 diabetes mellitus (T2DM). However, few studies, and none in Korea, have examined prevalence of T2DM and other variables in PWS. The aim of this study was to identify the prevalence and associated risk factors for T2DM in Korean patients with PWS.

Methods: We performed a retrospective cohort study of the 84 PWS patients aged 10 or over (10.3-35.8 years of age) diagnosed with PWS at Samsung Medical Center from 1994 to 2016. We estimated occurrence of T2DM according to age (10-18 years versus $>18$ years), body mass index (BMI), genotype, history of growth hormone therapy, homeostasis model of assessment-insulin resistance (HOMA-IR), and the presence of dyslipidemia, hypogonadism, or central precocious puberty. Additionally, we investigated cutoff values of risk factors for development of T2DM.

Results: Twenty-nine of a total 211 patients, diagnosed with PWS over the study period, were diagnosed as having T2DM (13.7\%, mean age $15.9 \pm 3.6$ years). In the $>18$ years group, obesity, HOMA-IR, and presence of dyslipidemia, hypogonadism, or central precocious puberty were associated with the occurrence of T2DM in univariate analysis. In multivariate logistic regression analysis, only obesity $(p=0.001)$ and $\operatorname{HOMA-IR}(p<0.001)$ were significant predictive factors for T2DM. Based on the receiver operating a characteristic curve analysis, the cutoff values of HOMA-IR and BMl for predicting T2DM were $>2.7$ and $>28.49 \mathrm{~kg} / \mathrm{m}^{2}$, respectively. Of the 29 patients, seven had $\geq 1$ microvascular complication, with non-proliferative diabetic retinopathy in 6 of 7 cases. Advanced age and HOMA-IR were positively correlated with diabetic microvascular complications ( $p<0.05$, Spearman correlation coefficient 0.393 and 0.434 , respectively).

Conclusions: The prevalence of diabetes in Korean PWS was similar to that in previous results. BMI and HOMA-IR were strong predictive factors for the development of T2DM in PWS. We specifically suggest the regular monitoring of glucose homeostasis parameters through a detailed settlement of ethnically specific cutoff values for BMI and HOMA-IR in PWS to prevent progression of T2DM and diabetic microvascular complications.
\end{abstract}

Keywords: Prader-Willi syndrome, Diabetes mellitus, Insulin, homeostasis model of assessment-insulin, Obesity

\section{Background}

Prader-Willi syndrome (PWS) is a contiguous gene syndrome that results from a lack of the expression of paternal alleles in the PWS region of chromosome 15q11-13 [1]. The clinical manifestations of PWS include hypotonia, early childhood-onset hyperphagia, characteristic facial appearance, hypogonadism, growth hormone deficiency, mild-to-severe mental retardation, and behavioral disturbance [2].

\footnotetext{
* Correspondence: nadri1217@naver.com; jindk@skku.edu Department of Pediatics, Samsung Medical Center, Sungkyunkwan University

School of Medicine, 81 Irwon-ro, Gangnam-gu, Seoul 06351, South Korea
}

Although patients with PWS show poor feeding and failure to thrive until nine months of age, they tend to be obese after then due to hypothalamic pituitary dysregulation-induced hyperphagia with a lack of satiety. This can lead to severe obesity in childhood [3], which often progressively develops into type-2 diabetes mellitus (T2DM), which is eventually associated with increased morbidity and mortality in PWS. In addition, uncontrolled DM-induced microvascular complications such as diabetic retinopathy, neuropathy, and nephropathy further impair quality of life in PWS. 
Obesity and insulin resistance are known to increase the risk of developing T2DM [4]; however, the occurrence of T2DM can be explained by multifactorial mechanisms, and the causal relationship between obesity and diabetes remains unclear. Moreover, obesity itself might not be a decisive factor of diabetes in PWS, considering the low visceral fat distribution and relative low insulin resistance compared to obese individuals without PWS [5]. The etiology of the development of T2DM in PWS has not yet been clarified and thus further research is warranted.

Previous literature places the prevalence of T2DM in PWS at approximately 7-24\% [6]. However, there has been no investigation of T2DM associated with PWS in Korea. Against the dramatically increasing incidence of T2DM in the general population, there are few studies available that are related to T2DM in PWS compared to the number of newly updated studies that have been conducted on T2DM in nonsyndromic population. Moreover, the cutoff values of HOMA-IR and BMI for T2DM are differentiated by race and ethnicity; in particular, the cutoff value for BMI in Asia is practically regarded as $23 \mathrm{~kg} / \mathrm{m}^{2}$. Thus, population and nationspecific studies are essential even in syndromic patients including PWS [7]. In this regard, additional large-scale elaborate investigations are required to identify the precise mechanism of diabetes in PWS besides establishing the primary and secondary prevention of T2DM. The present study estimated the prevalence of T2DM in Korean patients with PWS and attempted to identify the risk factors related to T2DM.

\section{Methods}

\section{Patients}

This study was approved by the institutional review board at Samsung Medical Center (2017-02-144). We reviewed the charts of 211 patients with PWS confirmed via methylation PCR between March 1994 and August 2016 at Samsung Medical Center. Among them, we selected patients aged 10 or older because the youngest patient with T2DM was 10.1 years old, and we compared two groups by dividing subjects into diabetic and nondiabetic groups. We excluded patients with previous bariatric surgery, chronic kidney disease, or no medical records resulted from a lack of clinic visits within the preceding year.

Overall, 84 subjects aged 10.3-35.8 years (i.e., born 1980-2005) were included (Fig. 1). All subjects were Korean individuals with PWS who had visited the hospital at least every six months for a regular checkup. All subjects had homeostasis model assessmentestimated insulin resistance (HOMA-IR) results and body gauge measurement within one year.

Besides, screening for microvascular complications such as comprehensive eye examination by an ophthalmologist,

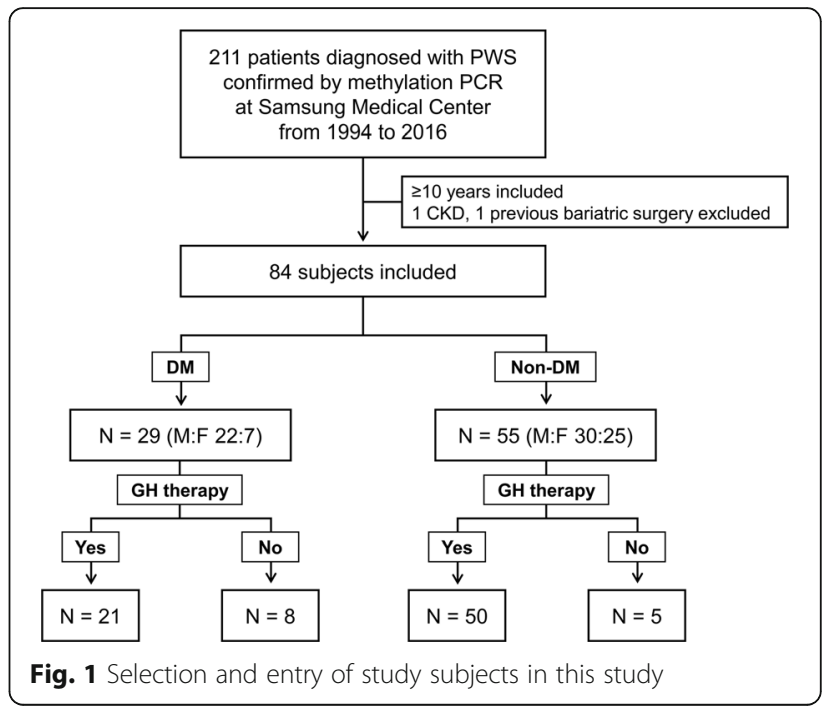

24-h urine collection with creatinine and microalbumin, and screening for autonomic neuropathy were performed at least annually in PWS patients with T2DM, including at the time of diagnosis.

\section{Anthropometric and laboratory measurements}

We used anthropometric variables measured by same standardized stadiometer only in the pediatric outpatient clinic of the Samsung medical center. Height was measured to the nearest $0.1 \mathrm{~cm}$ with a wall-mounted stadiometer (GL-310P, G-tech international, Uijungbu, Korea) and weight was determined to the nearest $0.1 \mathrm{~kg}$ on a medical scale by a trained person. Body mass index (BMI) was calculated as weight $(\mathrm{kg})$ divided by height (m) squared. BMI, and height and weight standard deviation scores (SDS) were calculated using the 2007 Korean children and adolescents growth standard [4].

Obesity was defined as the BMI cut-off point $>2$ SDS in children and adolescent patients (0-18 years) and BMI > 30 in adults [8]. Severe obesity was defined as having a $\mathrm{BMI} \geq 120 \%$ of the 95 th percentile or an absolute BMI $\geq 35 \mathrm{~kg} / \mathrm{m}^{2}$, whichever was lower based on age and gender [9]. Serum insulin concentrations were measured by immunoradiometric assay using an INS-IRMA kit (BioSource, Nivelles, Belgium).

Peripheral blood samples were obtained after a $12 \mathrm{~h}$ overnight fast (at least an eight-hour), and all laboratory data were analyzed at the Samsung medical center. Serum glucose was measured by the hexokinase/glucose-6-phosphate dehydrogenase method. Hemoglobin A1c (HbA1C) expressed as \% was measured by highperformance liquid chromatography.

Insulin resistance was measured using HOMA-IR, and calculated as follows: HOMA-IR = fasting insulin $(\mu \mathrm{U} /$ $\mathrm{mL}) \times$ fasting glucose $(\mathrm{mg} / \mathrm{dL}) / 22.5$ [10]. The patients were diagnosed with T2DM according to the American 
Diabetes Association (ADA) criteria as follows [11]: 1) a HbA1c level $\geq 6.5 \%$ or 2 ) a fasting blood sugar $($ FBS $) \geq 126 \mathrm{mg} / \mathrm{Dl}(7.0 \mathrm{mmol} / \mathrm{L})$ or 3$)$ a 2 -h postprandial plasma blood sugar $\geq 200 \mathrm{mg} / \mathrm{dL}$ during a $75 \mathrm{~g}$ oral glucose tolerance test (OGTT) or 4) a random plasma glucose $\geq 200 \mathrm{mg} / \mathrm{dL}$ in a patient with classic symptoms of hyperglycemia or hyperglycemic crisis.

Diabetic nephropathy (DN) is defined by proteinuria $>300 \mathrm{mg}$ for $24 \mathrm{~h}$ in the setting of diabetes, and microalbuminuria is defined as an albumin excretion of 30-299 mg/24 h.

Diabetic peripheral neuropathy (DPN) was screened for using a Neurometer ${ }^{ø}$ Current Perception Threshold $(\mathrm{CPT}) / \mathrm{C}$ (Neurotron Inc., Denver, CO, USA) in an environment-controlled room. The Neurometer ${ }^{\circledR} \mathrm{CPT} / \mathrm{C}$ is an electric current generator that provided selective stimulation for three sub-populations of sensory nerve fibers in the skin. The nerve is defined as normal if the CPT is in the normal range, the nerve is defined as in hyperesthesia if the CPT is below its normal range, and the nerve is defined as in hypoesthesia if the CPT is higher than the normal range. The methods of diabetic cardiac autonomic neuropathy assessment include the assessment of symptoms, signs, and cardiovascular autonomic reflex tests based on heart rate and BP variation to deep breathing, Valsalva maneuver, and postural change using the DICAN $^{\bullet}$ evaluation system (Medicore Co., Ltd., Seoul, Korea). DPN was defined as when abnormalities were found in both the CPT and autonomic function test.

\section{Statistical analysis}

The baseline characteristics were described using percentages for categorical variables and means \pm SD or mean changes (SD) for continuous variables. Comparisons between diabetic and non-diabetic groups were performed using the Wilcoxon-Mann-Whitney test for categorical variables and Student's t test for continuous variables. Univariate logistic regression analysis was employed to evaluate the crude effects of the variables on the development of T2DM. Variables associated $(p<0.10)$ with outcomes in the univariate analysis were subject to a multivariate backward stepwise logistic regression analysis to estimate their adjusted effects on the development of T2DM. The odds ratio (OR) and the 95\% confidence intervals (CIs) were computed from the regression analyses.

The impact of risk factors for T2DM were evaluated using areas under the curve for sensitivity and specificity, which were constructed (receiver-operator characteristic [ROC] curve [12]) using MedCalc version 17.4 (MedCalc Software, Ostend, Belgium). The ROC curve is a graphical representation of the relationship between sensitivity and specificity based on various anthropometric cut-off values.
A perfect test will have an AUC of 1.0, and AUC $=0.5$ means that the test performs no better than chance. The optimal cut-off point was identified based on the maximal Youden index (sensitivity + specificity -1 ).

Spearman rank order correlation was performed to evaluate possible correlations between microvascular complications and independent variables. All statistical analyses were performed using SPSS 23 (IBM Corporation, USA). A $p$ value of $<0.05$ was considered statistically significant.

\section{Results}

The baseline characteristics of all subjects above 10 years old with PWS are shown in Table 1. Out of the 211 diagnostically confirmed PWS patients, T2DM was found in 29 (13.7\%). The mean age at DM diagnosis was 15.9 years (10.1-27.0). The diabetic group was $0.4 \pm 5.7$ years older than the non-diabetic group (15.8 \pm 4.1 years). Although the percentage of males in the diabetic group (75.9\%), was slightly higher than the non-diabetic group (54.5\%), no significant sex ratio distribution was evident. The mean BMI in the diabetic group was $35.7 \pm 9.2 \mathrm{~kg} / \mathrm{m}^{2}$, corresponding to $2.8 \pm 1.0 \mathrm{SD}$, higher than the nondiabetic group $\left(28.3 \pm 8.9 \mathrm{~kg} / \mathrm{m}^{2}\right)$. Twenty-three patients among the $29(79.3 \%)$ in the diabetic group were obese, which was higher than the non-diabetic group (22/55, $40 \%)$. The mean HOMA-IR was $6.3 \pm 3.5$ in the diabetic group, which was higher than in the non-diabetic group (3.4 \pm 3.8$)$. Twenty-one $(72.4 \%)$ patients in the diabetic group and 50 patients (90.9\%) in the non-diabetic group had a history of GH treatment. However, the duration of $\mathrm{GH}$ treatment prior to the occurrence of T2DM was not significantly different between the diabetic and nondiabetes groups $(p=0.140)$. Meanwhile, the percentage of patients with dyslipidemia taking medications and the patients with hypogonadism who underwent hormone replacement therapy were higher than in the nondiabetic group, at $55.2 \%$ and $65.5 \%$, respectively (vs. $20.0 \%$ and $36.4 \%$ in the non-diabetic group). The proportion of patients with severe obesity was higher in PWS-DM group compared to the 84 subjects in total $(p<0.03)$ (Additional file 1: Fig. S1).

Regression analysis for the prevalence of T2DM was as shown in Table 2. Age above 18 years, obesity, HOMAIR, dyslipidemia, and hypogonadism were significant risk factors related with T2DM. Growth hormone treatment (GHT) was not a leading cause for the development of T2DM regardless of both age at GHT start and treatment period. In addition, neither genotype nor gender was associated with the development of T2DM. In multivariate analysis after covariation of factors (age, gender, BMI, HOMA-IR), HOMA-IR (OR 1.73, 95\% CI 1.31-2.29) and obesity (OR 6.76, 95\% CI 2.10-21.70) were significant predictors of T2DM development. 
Table 1 Baseline characteristics of the patients with PWS

\begin{tabular}{|c|c|c|c|c|}
\hline & $\begin{array}{l}\text { DM, n (\%) } \\
29(34.5)\end{array}$ & $\begin{array}{l}\text { Non-DM, n (\%) } \\
55(65.5)\end{array}$ & $\begin{array}{l}\text { Total, n (\%) } \\
84(100)\end{array}$ & $p$-value \\
\hline Age (years) (range) & $20.4 \pm 5.7(12.7-35.8)$ & $15.8 \pm 4.1(10.3-31.8)$ & $17.4 \pm 5.1(10.3-35.8)$ & $<0.001$ \\
\hline Age at diagnosis with DM (years) & $15.9 \pm 3.6(10.1-27.0)$ & - & - & - \\
\hline Age at diagnosis with PWS (years) & $6.9 \pm 7.2$ & $4.7 \pm 5.0$ & $5.5 \pm 5.9$ & 0.151 \\
\hline Gender (male), n (\%) & $22(75.9)$ & $30(54.5)$ & $52(61.9)$ & 0.057 \\
\hline Genotype (deletion), n (\%) & $21(72.4)$ & $38(69.1)$ & $59(70.2)$ & 0.757 \\
\hline BMl, kg/m2 (SD) & $35.7 \pm 9.2(2.8 \pm 1.0)$ & $28.3 \pm 8.9(1.7 \pm 1.3)$ & $30.8 \pm 9.6(2.1 \pm 1.3)$ & $<0.001$ \\
\hline Obesity $^{a}, \mathrm{n}(\%)$ & $23(79.3)$ & $22(40)$ & $57(67.9)$ & 0.001 \\
\hline HOMA-IR & $6.3 \pm 3.5$ & $3.4 \pm 3.8$ & $4.4 \pm 3.9$ & 0.001 \\
\hline $\mathrm{HbA1C}, \%(\mathrm{mmol} / \mathrm{mol})$ & $8.4 \pm 2.3$ & $5.6 \pm 0.3$ & $6.6 \pm 1.9$ & $<0.001$ \\
\hline Basal insulin $(\mu \mid \mathrm{U} / \mathrm{mL})$ & $45.7 \pm 31.1$ & $25.3 \pm 23.0$ & $32.5 \pm 27.7$ & 0.001 \\
\hline Basal C-peptide (ng/mL) & $7.0 \pm 2.8$ & $4.8 \pm 2.9$ & $5.7 \pm 3.0$ & 0.004 \\
\hline Age at GHT start (years) & $7.0 \pm 3.8$ & $6.7 \pm 4.3$ & $6.8 \pm 4.1$ & 0.795 \\
\hline Previous GHT duration (years) & $6.3 \pm 3.3$ & $7.5 \pm 3.1$ & $7.2 \pm 3.2$ & 0.140 \\
\hline GHT, n (\%) & $21(72.4)$ & $50(90.9)$ & $71(84.5)$ & 0.027 \\
\hline Dyslipidemia, n (\%) & $16(55.2)$ & $11(20.0)$ & $27(32.1)$ & 0.001 \\
\hline Hypothyroidism, n (\%) & $0(0)$ & $2(3.6)$ & $2(2.4)$ & 0.988 \\
\hline Hypogonadism, n (\%) & $19(65.5)$ & $20(36.4)$ & $39(46.4)$ & 0.011 \\
\hline Central precocious puberty, n (\%) & $2(6.9)$ & $12(21.8)$ & $14(16.7)$ & 0.071 \\
\hline
\end{tabular}

Data are presented as number (\%) or mean \pm SD

DM Diabetes Mellitus, DMT treatment of DM, GHT growth hormone treatment, BMI body mass index, HOMA-IR homeostasis model assessment, $H b A 1 C$ hemoglobin A1C *Significant at $p<0.05$

${ }^{a}$ In this study, obesity is defined as BMI cut-off point $>30$ in adults, $>2$ SDS in children and adolescents

Figure 2 shows the ROC curves for detecting T2DM using HOMA-IR, BMI $\left(\mathrm{kg} / \mathrm{m}^{2}\right)$, and BMI (SDS). The areas under the ROC curves were 0.843 (95\% CI: 0.7580.927), 0.765 (95\% CI: $0.660-0.851)$, and 0.757 (95\% CI: $0.652-0.844)$, respectively $(p<0.0001)$. HOMA-IR and
BMI (SDS and $\mathrm{kg} / \mathrm{m}^{2}$ ) were similar, but HOMA-IR was slightly better than BMI at detecting T2DM. The cut-off values for anthropometric indices determined using ROC analysis are summarized in Table 3 . The probability of T2DM was increased for HOMA-IR results $>2.7$

Table 2 Univariate and multivariate logistic regression analyses of variables associated with risk of T2DM in PWS

\begin{tabular}{|c|c|c|c|c|}
\hline \multirow[t]{2}{*}{ Variables } & \multicolumn{2}{|l|}{ Univariate analysis } & \multicolumn{2}{|c|}{ Multivariate analysis } \\
\hline & OR $(95 \% \mathrm{Cl})$ & $P$-value ${ }^{*}$ & OR (95\% Cl) & $P$-value \\
\hline Age (>18 years) & $6.81(2.51-18.47)$ & $<.001$ & $1.87(0.47-7.47)$ & 0.373 \\
\hline Gender (male) & $0.38(0.14-1.04)$ & 0.060 & $0.60(0.16-2.28)$ & 0.455 \\
\hline Genotype (deletion) & $0.85(0.30-2.40)$ & 0.756 & - & - \\
\hline Obesity $^{\mathrm{a}}\left(\mathrm{BMI}>2 \mathrm{SD}\right.$ or $>30 \mathrm{~kg} / \mathrm{m}^{2}$ ) & $5.75(2.02-16.40)$ & 0.001 & $6.76(2.10-21.70)$ & 0.001 \\
\hline HOMA-IR & $1.31(1.09-1.59)$ & 0.005 & $1.73(1.31-2.29)$ & $<.001$ \\
\hline Dyslipidemia (yes) & $4.92(1.84-13.20)$ & 0.002 & $1.55(0.38-6.33)$ & 0.545 \\
\hline GHT (yes) & $0.39(0.11-1.37)$ & 0.141 & - & - \\
\hline Age at GHT start (years) & $1.02(0.90-1.15)$ & 0.792 & - & - \\
\hline Duration of GHT ( $\geq 7.2$ years) & $0.75(0.27-2.07)$ & 0.572 & - & - \\
\hline Hypogonadism (yes) & $3.33(1.30-8.53)$ & 0.012 & $2.15(0.61-7.63)$ & 0.237 \\
\hline Central precocious puberty (yes) & $0.27(0.06-1.28)$ & 0.10 & - & - \\
\hline
\end{tabular}

Variables with $p<0.10$ were included in a multivariate logistic regression with conditional backward selection model

Abbreviations: T2DM type 2 Diabetes Mellitus, PWS Prader-Willi syndrome, OR odds ratio, Cl confidence interval, GHT growth hormone treatment, HOMA-IR homeostasis model assessment, $B M I$ body mass index

"Significant association was classified as $p<0.05$

${ }^{a}$ Obesity is defined as BMI cut-off point $>30$ in adults, $>2$ SDS in children and adolescents 


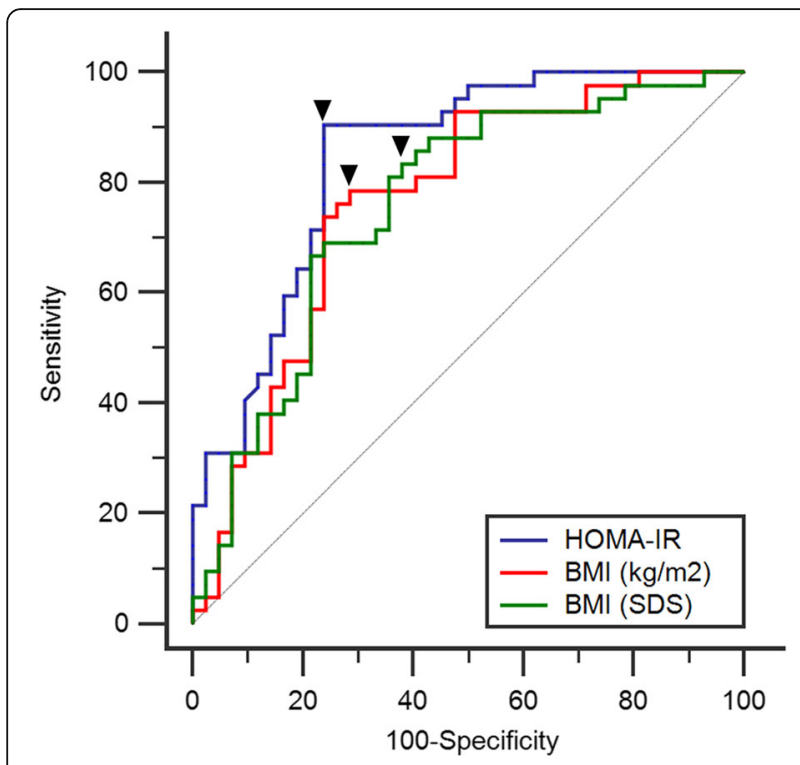

Fig. 2 Receiver-Operator Characteristic (ROC) curves predicting the impact of HOMA-IR (blue line), BMI (SDS) (green line), and BMI $\left(\mathrm{kg} / \mathrm{m}^{2}\right)$ (red line) on T2DM in PWS. The black line represents the zero discrimination line. Each black triangle denotes the point of cut-off value

(Youden's index 0.67), BMI $\left(\mathrm{kg} / \mathrm{m}^{2}\right)$ result $>28.49$ (Youden's index 0.50), and BMI (SDS) result $>1.73$ (Youden's index 0.45). These results were found to be suitable cut-off points for the detection of T2DM, as they possess the highest Youden's index.

Among the 29 patients with T2DM, seven showed microvascular complications. Spearman correlation analysis presented that the prevalence of microvascular complications for the occurrence of T2DM was positively associated with aging $(\mathrm{r}=0.393, p=0.047)$ and HOMAIR ( $\mathrm{r}=0.434, p=0.027)$. Meanwhile, the period of having T2DM tended to have a positive association with microvascular complications, but not to a significant degree $(\mathrm{r}=0.370, p=0.063)$ (Table 4).

Out of the seven patients with microvascular complications, nonproliferative retinopathy was found in five (17.2\%), microalbuminuria and DPN were shown in two (6.9\%) patients, and only one patient showed overt proteinuria (3.4\%) (Table 5). The time from the detection of

Table 3 Cut-off values for HOMA-IR, BMI $\left(\mathrm{kg} / \mathrm{m}^{2}\right)$, and BMI (SDS) as predictive values of T2DM development in PWS

\begin{tabular}{lllll}
\hline & Cut off point & $\begin{array}{l}\text { Area under the } \\
\text { curve }(95 \% \mathrm{Cl})\end{array}$ & $\begin{array}{l}\text { Sensitivity } \\
(95 \% \mathrm{Cl})\end{array}$ & $\begin{array}{l}\text { Specificity } \\
(95 \% \mathrm{Cl})\end{array}$ \\
\hline HOMA-IR & $2.7^{*}$ & 0.843 & 90.48 & 76.19 \\
$\mathrm{BMI}\left(\mathrm{kg} / \mathrm{m}^{2}\right)$ & $28.49^{*}$ & 0.765 & 78.57 & 71.43 \\
BMI (SDS) & $1.73^{*}$ & 0.757 & 83.33 & 61.90 \\
\hline
\end{tabular}

Abbreviations: T2DM type 2 Diabetes Mellitus, PWS Prader-Willi syndrome, HOMA-IR homeostasis model assessment, BMI body mass index, $\mathrm{Cl}$ confidence interval, $S D S$ standard deviation score

"Significant association was classified as $p<0.0001$
Table 4 Correlation analysis for microvascular complications and other variables in the diabetic group of PWS patients

\begin{tabular}{lll}
\hline Variables & \multicolumn{2}{l}{ Correlation coefficient } \\
\cline { 2 - 3 } & $r$ & $p$-value \\
\hline Age (years) & 0.393 & 0.047 \\
Age at diagnosis with PWS (years) & 0.226 & 0.267 \\
Age at diagnosis with T2DM (years) & 0.104 & 0.613 \\
HbA1C (\%) & 0.232 & 0.254 \\
BMI, kg/m2 (SD) & 0.133 & 0.517 \\
HOMA-IR & 0.434 & 0.027 \\
Age at GHT start (years) & 0.189 & 0.439 \\
Previous GHT duration (years) & 0.376 & 0.058 \\
DM treatment duration (years) & 0.370 & 0.063 \\
From PWS diagnosis to DM diagnosis (years) & -0.156 & 0.446 \\
\hline Abbrions: Span cort
\end{tabular}

Abbreviations: $r$ Spearman correlation coefficient, T2DM type 2 Diabetes Mellitus, PWS Prader-Willi syndrome, HbA1C hemoglobin A1C, GHT growth hormone treatment, HOMA-IR homeostasis model assessment, $B M I$ body mass index

"Significant association was classified as $p<0.05$

T2DM to these complications was different for each. One patient already had all three microvascular complications at the time of T2DM diagnosis. Notably, HbA1C upon the diagnosis of complications was higher than upon the diagnosis of T2DM. The BMI and HOMA-IR of seven patients with complications showed a tendency to be higher than those of other patients without complications.

\section{Discussion}

This is the first study about the prevalence and risk factors of T2DM in Korean PWS patients to our knowledge. The prevalence of T2DM in the present study was 13.7\% (29/ 211). This proportion is similar to that of previous studies (7-25\%) [13-16]. Considering the prevalence of T2DM in adults (6.1-6.9\%) [17] and $0.2 \%$ in children younger than 18 years in Korea [18], the prevalence rate of T2DM in Korean PWS is sufficiently high to warrant attention.

A recent Italian cohort study revealed a $13.5 \%$ prevalence of T2DM, which was similar to our results [15]. This proportion is relatively lower than the results of a Japanese study, which were $26.2 \%$ [16], but higher than in a French study, 0\% [19]. The differences in the described frequencies of T2DM have presumably resulted from various sizes of the PWS base population, the diagnostic approach to T2DM, the wide range of the age group, and the study period.

Insulin resistance and obesity are well known risk factors in T2DM. However, this relationship has been questioned in PWS due to relative hypoinsulinemia of PWS compared to non-syndromic obese individuals [20-22]. It seems that the reason for this considerably high insulin sensitivity is subcutaneous fat-dominant obesity with reduced visceral fat, higher plasma ghrelin 


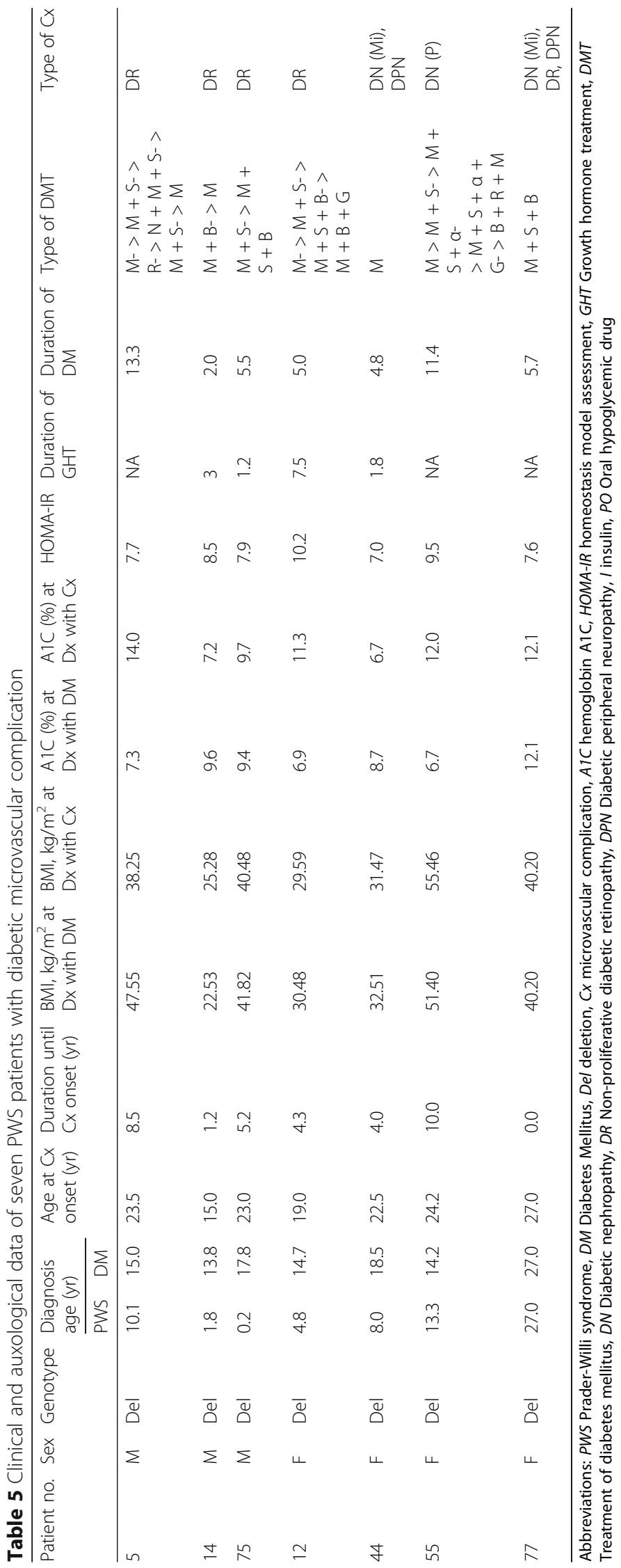


and adiponectin levels, reduced $\beta$-cell response to glucose stimulation, and insufficient growth hormone [23-26]. Acylated ghrelin is an orexigenic hormone that is associated with hyperphagia-induced obesity inducing positive energy balance and possibly involved in the development of diabetes in PWS [27]. However, although previous studies have not independently investigated T2DM, a recent study conducted in Italy [15] also showed that HOMA-IR and BMI were independent risk factors associated with T2DM in PWS, supporting our study result. Meanwhile, unlike the Italian results indicating that being $>18$ years of age was a significant risk factor for altered glucose metabolism (AGM), our result only indicated such significance in univariate analysis, not in multivariate analysis. This result can be explained by the distinction between study populations; 54 out of the 67 patients with AGM in the Italian study were over 18 years old and 52 were obese. In addition, it might have resulted from the difference in research targets, such as studying IFG and IGT, not only T2DM in the Italian study. Considering that age is generally a critical factor for the development of T2DM, the relatively younger age of our study group may be reflected in this outcome. In addition, it is noteworthy that the hypogonadism ratio, is significantly higher in the diabetic group than the non-diabetic group, in our study. Although obesity has been suggested as the important factor in decreased levels of total and free testosterone in T2DM, so far the relationship between hypogonadism and T2DM in PWS has not been clarified due to disease rarity and possibilities of multiple confounding factors [28]. However, our study results demonstrate a more potent approach to the relevance of diabetes and hypogonadism and further researches are needed. The present study also demonstrates that HOMA-IR and BMI have high sensitivity and specificity for assessing risk of T2DM with drawing cut off values through ROC curve analysis. The cutoff value of HOMA-IR of 2.7 for PWS with T2DM in our study is slightly higher than the cutoff value of 2.2-2.5 for metabolic syndrome in the general non-diabetic Korean population [29] and the cutoff value of 2.5 in the general non-diabetic Japanese population [30] but lower than the cutoff value of 3.16 for insulin resistance in Turkish children and adolescents without T2DM [31]. Meanwhile, compared to the cut off value of 4.0 for the diabetic group in Iranian patients [32], our result was considerably lower. While exact comparisons are difficult, the difference between these results suggests the possibility that patients with PWS are less resistant to insulin than non-syndromic patients with T2DM, which support previous research results that show quite high insulin sensitivity [33].

The cutoff value for BMI of $28.49 \mathrm{~kg} / \mathrm{m}^{2}$ in our study was lower than the cutoff value of $30 \mathrm{~kg} / \mathrm{m}^{2}$ in the general
Caucasian population, but it was higher than the cutoff value of less than $25 \mathrm{~kg} / \mathrm{m}^{2}$ in Asian population [7, 34, 35]. Our patients were all domestic Korean, which implies that PWS patients might be less likely to develop T2DM compared to non-syndromic population with similar BMI within the same race, which also lends credence to previous studies [5]. However, we need to take account of this influence on the cut off values by considering the differences in characteristics, sample size, study method, and gender in addition to ethnic and racial differences. Although there are no comparative studies on the sensitivity and specificity of the cutoff value for T2DM in PWS, these research attempts suggest that if precise large-scale research is conducted at a national level, it will be possible to increase the early diagnostic rate of T2DM in PWS and to take active measures for its management.

The causal relationship between $\mathrm{GH}$ therapy and the occurrence of T2DM has been a controversial issue for a long time. Recent studies have reached the conclusion that fasting insulin levels during GH therapy might be slightly elevated in children with PWS, but this is transient and does not eventually progress to diabetes [15, 36-39]. Furthermore, it is carefully suggested that if growth hormone is used at a low dose under strict metabolic control, it can increase muscle mass and insulin sensitivity while cutting down visceral fat and preventing the development of T2DM [40-42]. Still, there is no direct consensus for treatment target and dosage for central obesity to cut metabolic clusters. Moreover, given our study results, extreme obesity with a cut-off BMI value exceeding $26-28 \mathrm{~kg} / \mathrm{m}^{2}$ facilitates insulin secretion leading to overt diabetes via insulin resistance regardless of GH therapy, despite the effect of PWS [37, 43, 44].

In our study, age and HOMA-IR were found to be related to the occurrence of microvascular complications, but this study has limitations because it did not have a large number of subjects and was retrospective rather than a long-term prospective study. Several risk factors of microvascular complications have been reported in adults with T2DM, such as duration of diabetes, age, blood pressure, fasting plasma glucose, urinary albumin excretion levels, and elevated C-Reactive Protein levels [45-47]. However, it is difficult to compare our results because there have been few reports of complications related to T2DM in PWS [48]. The disease's rarity has meant that little research has been conducted despite the high prevalence of T2DM in PWS. Although not yet clear, ethnicity and familial insulin resistance characteristics may be associated with the development of diabetes in PWS, and further studies are expected to examine this.

Another limitations of this study are as follows: 1) The possibility that selection bias occurred in patients who have relatively good compliance with regular hospital follow-ups. 2) The possibility that the average age of 
study subjects might be lowered due to patients older than 30 years usually having poor compliance, thus being excluded from our study. 3) Failure to perform subcutaneous fat tissue comparison with dual-energy X-ray absorptiometry. 4) The 12-h fasting (at least 8-h) laboratory test was not performed perfectly due to the nature of PWS of intolerance to permanent hunger.

Nevertheless, the present study is worthwhile as the first in Korea on PWS patients diagnosed with T2DM. In addition, anthropometric measurements using the same method and stadiometer minimized measurement error, and a biochemical study conducted in the same laboratory made our results more reliable.

Several studies have reported that insulin resistance would not be significantly involved in the causes of T2DM in PWS due to the better insulin sensitivity of patients compared to obese individuals with PWS [5, 33]. However, according to recent studies including our results, although patients with PWS may have relative insulin sensitivity, insulin resistance and obesity are considered the most important factors in the occurrence of T2DM through the destruction of $\beta$-cell function $[15,16,37]$.

Our results support that obesity plays a major role in metabolic clusters in both children and adolescents with PWS. Therefore, it is necessary to actively control body weight through dietary control and exercise from early childhood to prevent severe obesity and T2DM.

\section{Conclusions}

In conclusion, the prevalence of T2DM in Korean PWS in our study was similar to the results of previous studies. BMI and HOMA-IR are strong predictive factors for the development of T2DM in PWS. In addition, our results suggest a relatively high cutoff level for the development of T2DM in PWS compared to non-syndromic obese control within the same race.

We emphasize early intervention to prevent severe obesity and the regular monitoring of glucose homeostasis parameters to predict the occurrence of T2DM in PWS. Further longitudinal studies are required to better understand the endocrine and metabolic factors that can determine T2DM development in PWS individuals.

\section{Additional file}

Additional file 1: Figure S1. Stratification of study population according to severity of obesity. Overweight: BMI cutoff point from 1.4 to 2 SDS in children and adolescents patients (0-18 yrs) and BMl from 25 to $30 \mathrm{~kg} / \mathrm{m}^{2}$ in adults; Obesity: BMl cut-off point $>2$ SDS (0-18 years) and $\mathrm{BMI}>30 \mathrm{~kg} / \mathrm{m}^{2}$ in adults; Severe obesity: BMI $\geq 120 \%$ of the 95 th percentile or an absolute BMI $\geq 35 \mathrm{~kg} / \mathrm{m}^{2}$ (TIFF $362 \mathrm{~kb}$ )

\section{Abbreviations}

AGM: Altered glucose metabolism; BMI: Body mass index; Cls: Confidence intervals; CPT: Current Perception Threshold; DN: Diabetic nephropathy; DPN: Diabetic peripheral neuropathy; GH: Growth hormone; GHT: Growth hormone treatment; HbA1C: Hemoglobin A1C; HOMA-IR: Homeostasis model assessment-estimated insulin resistance; OR: Odds ratio; PWS: Prader-Willi syndrome; ROC: Receiver-operator characteristic; SD: Standard deviation; SDS: Standard deviation score; T2DM: Type 2 diabetes mellitus

\section{Acknowledgements}

We thank all the individuals living with rare diseases and their families and all the clinical and research laboratory staff.

Funding

This study was supported by a grant from Samsung Medical Center (\#GFO217006).

Availability of data and materials

Not applicable.

\section{Authors' contributions}

AY designed and supervised the study, overseeing the data collection, interpretation, management, statistical analysis and drafting of the article for this study. JK was the study coordinator. He was responsible for the collection of clinical data and diagnostic information of PWS patients in our center. SYC contributed to the research design, data analysis and interpretation, the drafting and critical review of the paper, and the approval of the submitted paper. D-KJ was the team leader, secured funding for this project, and also contributed the approval of the submitted paper. SYC and $\mathrm{D}-\mathrm{KJ}$ contributed equally to this work. All authors read and approved the final manuscript.

\section{Ethics approval and consent to participate}

Written informed consents were obtained from patients and their parents of each patient, and the Institutional Review Board at Samsung Medical Center approved the study (IRB file number: 2017-02-144).

Consent for publication

Not applicable.

\section{Competing interests}

The authors declare that they have no competing interests.

\section{Publisher's Note}

Springer Nature remains neutral with regard to jurisdictional claims in published maps and institutional affiliations.

Received: 26 April 2017 Accepted: 23 August 2017

Published online: 30 August 2017

References

1. Bittel DC, Butler MG. Prader-Willi syndrome: clinical genetics, cytogenetics and molecular biology. Expert Rev Mol Med. 2005;7:1-20.

2. Cassidy SB, Driscoll DJ. Prader-Willi syndrome. Eur J Hum Genet. 2009:17:3-13.

3. Burman P, Ritzen EM, Lindgren AC. Endocrine dysfunction in Prader-Willi syndrome: a review with special reference to $\mathrm{GH}$. Endocr Rev. 2001:22:787-99.

4. Lim SM, Choi DP, Rhee Y, Kim HC. Association between obesity indices and insulin resistance among healthy Korean adolescents: the JS high school study. PLoS One. 2015;10:e0125238.

5. Goldstone AP, Thomas EL, Brynes AE, Bell JD, Frost G, Saeed N, et al. Visceral adipose tissue and metabolic complications of obesity are reduced in Prader-Willi syndrome female adults: evidence for novel influences on body fat distribution. J Clin Endocrinol Metab. 2001;86:4330-8.

6. Sinnema M, Maaskant MA, van Schrojenstein Lantman-de Valk HM, van Nieuwpoort IC, Drent ML, Curfs LM, et al. Physical health problems in adults with Prader-Willi syndrome. Am J Med Genet A. 2011;155a:2112-24.

7. Hsu WC, Araneta MRG, Kanaya AM, Chiang JL, Fujimoto W. BMI cut points to identify at-risk Asian Americans for type 2 diabetes screening. Diabetes Care. 2015:38:150-8.

8. Cole TJ, Bellizzi MC, Flegal KM, Dietz WH. Establishing a standard definition for child overweight and obesity worldwide: international survey. BMJ. 2000;320:1240. 
9. Koebnick C, Smith N, Coleman KJ, Getahun D, Reynolds K, Quinn VP, et al. Prevalence of Extreme Obesity in a Multiethnic Cohort of Children and Adolescents. The Journal of Pediatrics. 2010;157 26-31.e2

10. Matthews DR, Hosker JP, Rudenski AS, Naylor BA, Treacher DF, Turner RC. Homeostasis model assessment: insulin resistance and $\beta$-cell function from fasting plasma glucose and insulin concentrations in man. Diabetologia. 1985;28:412-9.

11. Classification and Diagnosis of Diabetes. Diabetes Care. 2016;39:S13-22.

12. Greiner M, Pfeiffer D, Smith RD. Principles and practical application of the receiver-operating characteristic analysis for diagnostic tests. Prev Vet Med. 2000;45:23-41.

13. Butler JV, Whittington JE, Holland AJ, Boer H, Clarke D, Webb T. Prevalence of and risk factors for, physical ill-health in people with Prader-Willi syndrome: a population-based study. Dev Med Child Neurol. 2002;44:248-55.

14. Zipf WB. Glucose homeostasis in Prader-Willi syndrome and potential implications of growth hormone therapy. Acta Paediatr Suppl. 1999:88:115-7.

15. Fintini D, Grugni G, Bocchini S, Brufani C, Di Candia S, Corrias A, et al. Disorders of glucose metabolism in Prader-Willi syndrome: results of a multicenter Italian cohort study. Nutr Metab Cardiovasc Dis. 2016;26:842-7.

16. Tsuchiya T, Oto Y, Ayabe T, Obata K, Murakami N, Nagai T. Characterization of diabetes mellitus in Japanese prader-willi syndrome. Clin Pediatr Endocrinol. 2011;20:33-8.

17. Koo BK, Lee $\mathrm{C}-\mathrm{H}$, Yang BR, Hwang S-S, Choi N-K. The Incidence and Prevalence of Diabetes Mellitus and Related Atherosclerotic Complications in Korea. A National Health Insurance Database Study PLOS ONE. 2014;9: e110650.

18. Ha KH, Kim DJ. Epidemiology of childhood obesity in Korea. Endocrinol Metab (Seoul). 2016;31:510-8

19. Diene G, Mimoun E, Feigerlova E, Caula S, Molinas C, Grandjean H, et al. Endocrine disorders in children with Prader-Willi syndrome-data from 142 children of the French database. Horm Res Paediatr. 2010;74:121-8.

20. Haqq AM, Muehlbauer MJ, Newgard CB, Grambow S, Freemark M. The metabolic phenotype of Prader-Willi syndrome (PWS) in childhood: heightened insulin sensitivity relative to body mass index. J Clin Endocrinol Metab. 2011;96:E225-32.

21. Hoybye C, Hilding A, Jacobsson H, Thoren M. Metabolic profile and body composition in adults with Prader-Willi syndrome and severe obesity. J Clin Endocrinol Metab. 2002:87:3590-7.

22. Krochik AG, Ozuna B, Torrado M, Chertkoff L, Mazza C. Characterization of alterations in carbohydrate metabolism in children with Prader-Willi syndrome. J Pediatr Endocrinol Metab. 2006;19:911-8.

23. Schuster DP, Osei K, Zipf WB. Characterization of alterations in glucose and insulin metabolism in Prader-Willi subjects. Metabolism. 1996;45:1514-20.

24. Khan MJ. Gerasimidis K. Shaikh MG. Mechanisms of obesity in Prader-Willi syndrome. Pediatr Obes: Edwards CA; 2016.

25. Kennedy L, Bittel DC, Kibiryeva N, Kalra SP, Torto R, Butler MG. Circulating adiponectin levels, body composition and obesity-related variables in Prader-Willi syndrome: comparison with obese subjects. Int J Obes (Lond). 2006;30:382-7

26. Cadoudal T, Buleon M, Sengenes C, Diene G, Desneulin F, Molinas C, et al. Impairment of adipose tissue in Prader-Willi syndrome rescued by growth hormone treatment. Int J Obes (Lond). 2014;38:1234-40.

27. Kuppens RJ, Diène G, Bakker NE, Molinas C, Faye S, Nicolino M, et al. Elevated ratio of acylated to unacylated ghrelin in children and young adults with Prader-Willi syndrome. Endocrine. 2015:50:633-42.

28. Betancourt-Albrecht M, Cunningham GR. Hypogonadism and diabetes. Int J Impot Res. 0000;15:S14-S20.

29. Yun KJ, Han K, Kim MK, Park YM, Baek KH, Song KH, et al. Insulin resistance distribution and cut-off value in Koreans from the 2008-2010 Korean National Health and nutrition examination survey. PLoS One. 2016;11:e0154593

30. Yamada C, Mitsuhashi T, Hiratsuka N, Inabe F, Araida N, Takahashi E. Optimal reference interval for homeostasis model assessment of insulin resistance in a Japanese population. J Diabetes Investig. 2011;2:373-6.

31. Keskin M, Kurtoglu S, Kendirci M, Atabek ME, Yazici C. Homeostasis model assessment is more reliable than the fasting glucose/insulin ratio and quantitative insulin sensitivity check index for assessing insulin resistance among obese children and adolescents. Pediatrics. 2005;115:e500-3.

32. Esteghamati A, Ashraf H, Khalilzadeh O, Zandieh A, Nakhjavani M, Rashidi A, et al. Optimal cut-off of homeostasis model assessment of insulin resistance (HOMA-IR) for the diagnosis of metabolic syndrome: third national surveillance of risk factors of non-communicable diseases in Iran (SuRFNCD2007). Nutr Metab (Lond). 2010;7:26.

33. Talebizadeh Z, Butler MG. Insulin resistance and obesity-related factors in Prader-Willi syndrome: comparison with obese subjects. Clin Genet. 2005:67:230-9.

34. Appropriate body-mass index for Asian populations and its implications for policy and intervention strategies. Lancet. 2004;363:157-63.

35. Chiu M, Austin PC, Manuel DG, Shah BR, Tu JV. Deriving ethnic-specific BMI cutoff points for assessing diabetes risk. Diabetes Care. 2011;34:1741-8.

36. Myers SE, Carrel AL, Whitman BY, Allen DB. Sustained benefit after 2 years of growth hormone on body composition, fat utilization, physical strength and agility, and growth in Prader-Willi syndrome. J Pediatr. 2000;137:42-9.

37. L'Allemand D, Eiholzer U, Schlumpf M, Torresani T, Girard J. Carbohydrate metabolism is not impaired after 3 years of growth hormone therapy in children with Prader-Willi syndrome. Horm Res. 2003;59:239-48.

38. Bakker NE, Kuppens RJ, Siemensma EP, Tummers-de Lind van Wijngaarden RF, Festen DA, Bindels-de Heus GC, et al. Eight years of growth hormone treatment in children with Prader-Willi syndrome: maintaining the positive effects. J Clin Endocrinol Metab. 2013;98:4013-22.

39. Hoybye C. Growth hormone treatment of Prader-Willi syndrome has longterm, positive effects on body composition. Acta Paediatr. 2015;104:422-7.

40. Nam SY, Kim KR, Cha BS, Song YD, Lim SK, Lee HC, et al. Low-dose growth hormone treatment combined with diet restriction decreases insulin resistance by reducing visceral fat and increasing muscle mass in obese type 2 diabetic patients. Int J Obes Relat Metab Disord. 2001;25:1101-7.

41. Holt R. Growth hormone: a potential treatment option in diabetes? Diabetes Voice. 2003;48:22-4

42. Johannsson G, Marin P, Lonn L, Ottosson M, Stenlof K, Bjorntorp P, et al. Growth hormone treatment of abdominally obese men reduces abdominal fat mass, improves glucose and lipoprotein metabolism, and reduces diastolic blood pressure. J Clin Endocrinol Metab. 1997;82:727-34

43. Campbell PJ, Gerich JE. Impact of obesity on insulin action in volunteers with normal glucose tolerance: demonstration of a threshold for the adverse effect of obesity. J Clin Endocrinol Metab. 1990;70:1114-8.

44. Salomon F, Cuneo RC, Umpleby AM, Sonksen PH. Interactions of body fat and muscle mass with substrate concentrations and fasting insulin levels in adults with growth hormone deficiency. Clin Sci (Lond). 1994;87:201-6.

45. Wandell PE. Risk factors for microvascular and macrovascular complications in men and women with type 2 diabetes. Scand J Prim Health Care. 1999;17:116-21.

46. Nazimek-Siewniak B, Moczulski D, Grzeszczak W. Risk of macrovascular and microvascular complications in type 2 diabetes: results of longitudinal study design. J Diabetes Complicat. 2002;16:271-6.

47. del Cañizo Gómez FJ, Fernández Pérez C, Moreno Ruiz I, de Gorospe P-JC, Silveira Rodríguez B, González Losada T, et al. Microvascular complications and risk factors in patients with type 2 diabetes. Endocrinol Nutr. 2011;58:163-8

48. Bassali R, Hoffman WH, Chen H, Tuck-Muller CM. Hyperlipidemia, insulindependent diabetes mellitus, and rapidly progressive diabetic retinopathy and nephropathy in Prader-Willi syndrome with del(15)(q11.2q13). Am J Med Genet. 1997:71:267-70.

\section{Submit your next manuscript to BioMed Central and we will help you at every step:}

- We accept pre-submission inquiries

- Our selector tool helps you to find the most relevant journal

- We provide round the clock customer support

- Convenient online submission

- Thorough peer review

- Inclusion in PubMed and all major indexing services

- Maximum visibility for your research

Submit your manuscript at www.biomedcentral.com/submit
) Biomed Central 